\title{
Coherent radio emission from a quiescent red dwarf indicative of star-planet interaction
}

\author{
H. K. Vedantham ${ }^{1,2}{ }^{4}$, J. R. Callingham ${ }^{1}{ }^{1}$, T. W. Shimwell ${ }^{1,3}$, C. Tasse ${ }^{4}$, B. J. S. Pope $\mathbb{1}^{5}$, M. Bedell $^{6}$, \\ I. Snellen 3 , P. Best7, M. J. Hardcastle ${ }^{8}{ }^{8}$, M. Haverkorn ${ }^{9}$, A. Mechev ${ }^{3}$, S. P. O'Sullivan ${ }^{10,11}$, \\ H. J. A. Röttgering $\oplus^{3}$ and G. J. White $e^{12,13}$
}

\begin{abstract}
Low-frequency $(\nu \lesssim 150 \mathrm{MHz})$ stellar radio emission is expected to originate in the outer corona at heights comparable to and larger than the stellar radius. Such emission from the Sun has been used to study coronal structure, mass ejections and space-weather conditions around the planets'. Searches for low-frequency emission from other stars have detected only a single active flare star ${ }^{2}$ that is not representative of the wider stellar population. Here we report the detection of low-frequency radio emission from a quiescent star, GJ 1151-a member of the most common stellar type (red dwarf or spectral class $M$ ) in the Galaxy. The characteristics of the emission are similar to those of planetary auroral emissions ${ }^{3}$ (for example, Jupiter's decametric emission), suggesting a coronal structure dominated by a global magnetosphere with low plasma density. Our results show that large-scale currents that power radio aurorae operate over a vast range of mass and atmospheric composition, ranging from terrestrial planets to main-sequence stars. The Poynting flux required to produce the observed radio emission cannot be generated by GJ 1151's slow rotation, but can originate in a sub-Alfvénic interaction of its magnetospheric plasma with a short-period exoplanet. The emission properties are consistent with theoretical expectations ${ }^{4-7}$ for interaction with an Earth-size planet in an approximately one- to five-day-long orbit.
\end{abstract}

We discovered radio emission in the direction of the quiescent red dwarf star GJ 1151 by cross-matching catalogued radio sources in the Low Frequency Array (LOFAR) Two-Metre Sky Survey (LoTSS) data release $\mathrm{I}^{8}$, with nearby stars within a distance of $d<20$ pc from the Gaia data release 2 database ${ }^{9}$. The distance cut was imposed to maximize our chances of finding inherently faint stellar and planetary radio emission while maintaining a low false association rate ${ }^{10}$. We found one match at high significance: GJ 1151 , which is the closest catalogued star within the radio survey footprint. The radio source lies at a distance of $0.17(55)^{\prime \prime}$ in right ascension and $0.63(45)^{\prime \prime}$ in declination from the proper-motioncorrected optical position of GJ 1151 ( $1 \sigma$ errors in parentheses hereafter; see Extended Fig. 1).

GJ 1151 was observed by four partially overlapping LoTSS pointings conducted within a span of about one month. The LoTSS radio source ILT J115055.50+482225.2 is detected in only one, and has a high circularly polarized fraction of $64 \pm 6 \%$ (Fig. 1). The transient nature and high polarization fraction are inconsistent with known properties of extragalactic radio sources, but consistent with that of stellar and planetary emissions ${ }^{11}$. On the basis of the positional co-incidence, transient nature and high circularly polarized fraction, we conclusively associate the radio source with GJ 1151. The astrometric uncertainty of $\sim 0.2^{\prime \prime}$ in LoTSS data is insufficient to astrometrically differentiate between the stellar corona and a hypothetical planetary magnetosphere as the site of emission.

To determine the spectro-temporal characteristics of the radio emission, we extracted its time-averaged spectrum and frequencyaveraged light curve (Methods). We found that despite temporal variability, the emission persisted for the entire $8 \mathrm{~h}$ observation. The emission is also detected over the entire available bandwidth, $120<\nu<167 \mathrm{MHz}$ (where $\nu$ is the observed frequency), and has an approximately flat spectral shape (Fig. 2). The in-band radio power for an isotropic emitter is $P_{\mathrm{R}} \approx 2 \times 10^{21} \mathrm{ergs} \mathrm{s}^{-1}$. The peak radiation brightness temperature is $T_{\mathrm{b}} \approx 3.7 \times 10^{12} x_{*}^{-2} \mathrm{~K}$ where $x_{*}$ is the radius of the emitter in units of GJ 1151's stellar radius $R_{*} \approx 1.3 \times 10^{10} \mathrm{~cm}$.

A unique aspect of this detected radio source is that it is associated to a star with a quiescent chromosphere. Stellar radio emission at gigahertz frequencies is predominately non-thermal in origin and is powered by chromospheric magnetic activity. The majority of stellar radio detections are of a small class of magnetically active stars such as flare stars ${ }^{12,13}$ (for example, AD Leo), rapid rotators ${ }^{14}$ (for example, FK Com) and close binaries ${ }^{15}$ (for example, Algol). GJ 1151, in contrast, is a canonical 'quiescent' star, such as the Sun, based on all available chromospheric activity indicators (Table 1). For comparison, relatively intense broadband noise storms on the Sun are arcmin-scale sources with brightness temperatures of $T_{\mathrm{b}} \approx 10^{9} \mathrm{~K}\left(\right.$ ref. $\left.{ }^{16}\right)$. Such an emitter will be three orders of magnitude fainter than the radio source in GJ 1151 if observed from the same distance.

In addition to the quiescent nature of GJ 1151, the properties of the observed radio emission are distinct from prototypical stellar bursts at centimetre wavelengths. Stellar radio emission falls into two broad phenomenological categories ${ }^{11}$. (1) Incoherent gyrosynchrotron emission, similar to solar noise storms ${ }^{16}$, characterized by a low degree of polarization, brightness temperatures of $T_{\mathrm{b}} \lesssim 10^{10} \mathrm{~K}$,

\footnotetext{
'ASTRON, Netherlands Institute for Radio Astronomy, Dwingeloo, The Netherlands. ${ }^{2}$ Kapteyn Astronomical Institute, University of Groningen, Groningen, The Netherlands. ${ }^{3}$ Leiden Observatory, Leiden University, Leiden, The Netherlands. ${ }^{4}$ GEPI, Observatoire de Paris, Université PSL, CNRS, Meudon, France. ${ }^{5}$ NASA Sagan Fellow, Center for Cosmology and Particle Physics, Department of Physics, New York University, New York, NY, USA. ${ }^{6}$ Flatiron Institute, Simons Foundation, New York, NY, USA. ${ }^{7}$ Institute for Astronomy, Royal Observatory, Edinburgh, UK. ${ }^{8}$ Centre for Astrophysics Research, University of Hertfordshire, Hatfield, UK. ${ }^{9}$ Radboud University Nijmegen, Nijmegen, The Netherlands. ${ }^{10}$ Hamburger Sternwarte, Universität Hamburg, Hamburg, Germany. " 11 chool of Physical Sciences and Centre for Astrophysics and Relativity, Dublin City University, Glasnevin, Ireland. ${ }^{12}$ Department of Physics and Astronomy, The Open University, Milton Keynes, UK. ${ }^{3}$ RAL Space, STFC Rutherford Appleton Laboratory, Didcot, UK. $\varpi_{e}$-mail: vedantham@astron.nl
} 


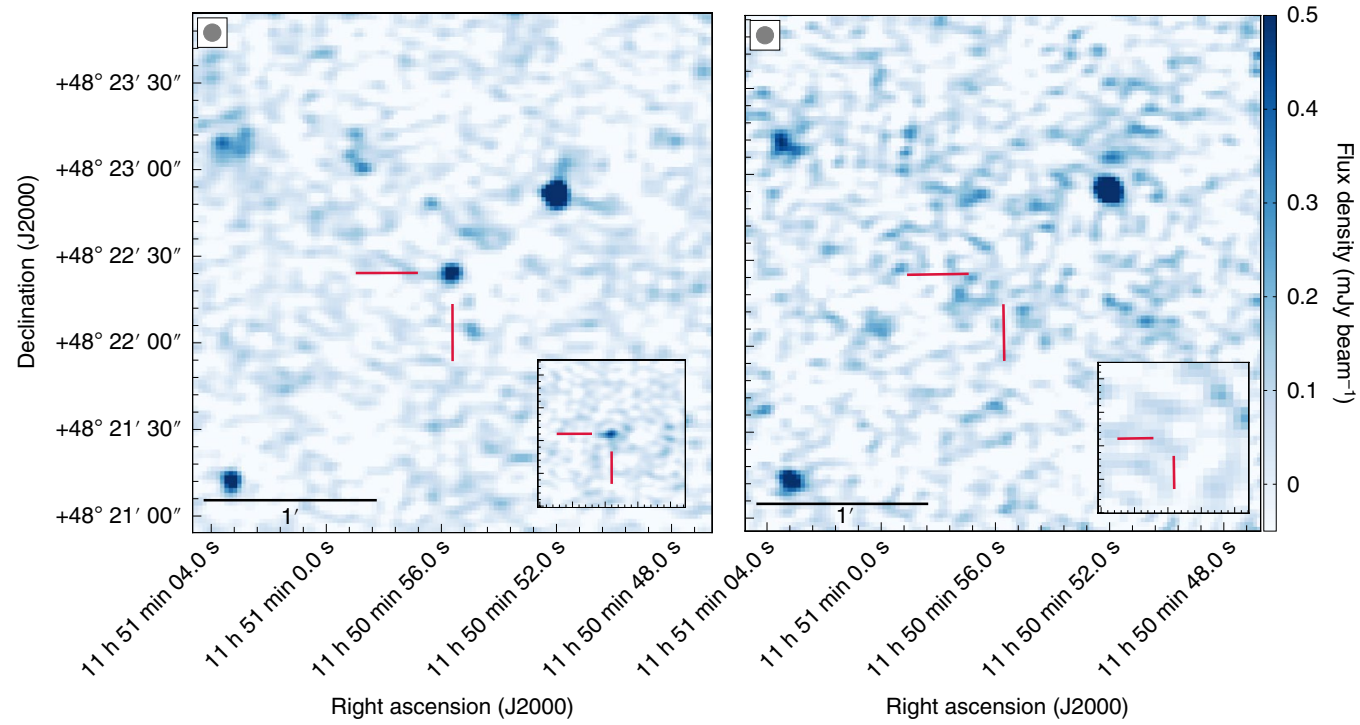

Fig. 1 | Total intensity deconvolved images of the region around GJ 1151 for two different epochs. Left panel: 16 June 2014 . Right: 28 May 2014 . The crosshairs point to the location of GJ 1151 (see Extended Fig. 1 for astrometric details). The inset in both panels displays the Stokes V (circular polarization) image for the respective epoch. The time-frequency-averaged Stokes I and $\vee$ flux densities are 0.89(8) mJy and 0.57(4) mJy, respectively. The grey circle in top-left corner indicates the width of the point spread function.
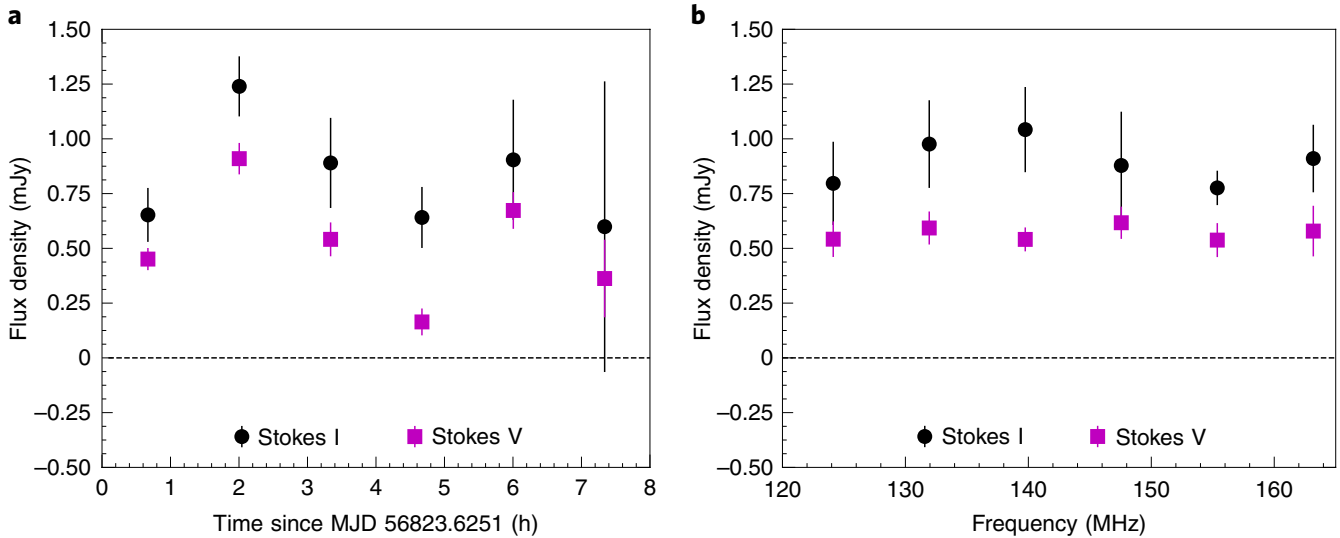

Fig. 2 | The variability of the flux density. a,b, The temporal (a) and spectral (b) variability of the total flux density (Stokes l; black circles) and circular polarized flux density (Stokes V; magenta squares) of the radio source in GJ 1151. The spectrum is measured over the entire $8 \mathrm{~h}$ exposure and the time series is measured over the entire bandwidth. The error bars span $\pm 1 \sigma$. MJD is the modified Julian date.

bandwidths of $\Delta \nu / \nu \sim 1$ and a duration of many hours. (2) Coherent emission (plasma or cyclotron emission), similar to solar radio bursts, characterized by a high degree of circular polarization (up to $100 \%)$, narrow instantaneous bandwidths $(\Delta \nu / \nu \ll 1)$ and a duration ranging from seconds to minutes. The observed emission does not fit into either of these phenomenological classes. It is broadband, has a duration of $>8 \mathrm{~h}$ and is highly circularly polarized. The closest analogue of such emission is auroral radio emission from substellar objects such as planets and ultracool dwarfs ${ }^{3,17,18}$. While canonical stellar radio bursts are powered by impulsive heating of plasma trapped in compact coronal loops ${ }^{11,19}$ of size much smaller than the stellar radius, radio aurorae in substellar objects are driven by global current systems in a large-scale dipolar magnetic field.

To gain further insight into the nature of the emission, we constrained the physical properties of the radio source from first principles. The high brightness temperature and high polarization fraction require the emission to originate from a coherent emission mechanism. The two known classes of coherent emission in non- relativistic plasma are plasma and cyclotron emission, which lead to emission at harmonics of the plasma frequency $\nu_{\mathrm{p}}$ and the cyclotron frequency $\nu_{c}$, respectively.

Stellar busts at centimetre wavelengths have previously been successfully modelled as fundamental plasma emission from coronal loops ${ }^{19}$. However, the emissivity of the fundamental emission drops nonlinearly with decreasing frequency. For typical coronal scale heights of quiescent red dwarfs, the height-integrated fundamental emission is restricted to brightness temperatures of $<10^{11} \mathrm{~K}$ at $150 \mathrm{MHz}$ (Methods), which cannot account for the observed emission with $T_{\mathrm{b}} \sim 10^{12} \mathrm{~K}$. Second harmonic plasma emission has a higher emissivity at low frequencies but cannot attain the high observed level of fractional polarization (Methods). These inconsistencies lead us to reject plasma emission as the cause and conclude that we are observing cyclotron maser emission.

Cyclotron maser emission occurs at harmonics of the local cyclotron frequency of $\nu_{\mathrm{c}} \approx 2.8 \mathrm{~B} \mathrm{MHz}$, where $B$ is the magnetic field strength in gauss. It is many orders of magnitude more efficient 


\begin{tabular}{|c|c|c|}
\hline Parameter & GJ 1151 & AD Leo \\
\hline Spectral type & M4.5V & M3V \\
\hline Distance (pc) & 8.04 & 4.965 \\
\hline Mass $\left(M_{\odot}\right)$ & $0.17\left(\right.$ ref. $\left.{ }^{36}\right)$ & $0.42\left(\right.$ ref. $^{36}$ ) \\
\hline Radius $\left(R_{\odot}\right)$ & $0.2\left(\right.$ ref. ${ }^{36}$ ) & $0.43\left(\right.$ ref. ${ }^{36}$ ) \\
\hline H $\alpha$ equivalent width $(\AA)$ & $0.034 \pm 0.041\left(\right.$ ref. $\left.^{36}\right)$ & $-3.311 \pm 0.017\left(\right.$ ref. $\left.^{36}\right)$ \\
\hline $\begin{array}{l}\mathrm{H} \alpha / \text { bolometric } \\
\text { luminosity }\left(\times 10^{-4}\right)\end{array}$ & $0.067\left(\right.$ ref. $\left.^{36}\right)$ & $1.72\left(\right.$ ref. $\left.^{36}\right)$ \\
\hline $\begin{array}{l}\text { ROSAT X-ray luminosity } \\
\left(\times 10^{28} \mathrm{erg} \mathrm{s}^{-1}\right)\end{array}$ & $<0.016\left(\right.$ ref. $\left.{ }^{37}\right)$ & $9.2 \pm 0.5\left(\right.$ ref. $\left.^{38}\right)$ \\
\hline $\begin{array}{l}\text { ROSAT X-ray/bolometric } \\
\text { luminosity }\left(\times 10^{-5}\right)\end{array}$ & $<1.07\left(\right.$ ref. ${ }^{37}$ ) & 105.74 (ref. ${ }^{39}$ ) \\
\hline Rotation period (d) & $125 \pm 23\left(\right.$ ref. $\left.^{40}\right)$ & $2.23\left(\right.$ ref. $\left.^{41}\right)$ \\
\hline $\begin{array}{l}\text { Coronal magnetic field } \\
\text { strength }(\mathrm{kG})\end{array}$ & Unknown & $0.19\left(\right.$ ref. $\left.^{42}\right)$ \\
\hline
\end{tabular}

The reported Röntgen Satellite (ROSAT) luminosities band is for the 0.1-2.4 keV band. All X-ray luminosities are reported for non-flare states. The upper limits reported on the X-ray luminosity of GJ 1151 are derived from $3 \sigma$ upper bound on the source flux. Distances are derived from Gaia data

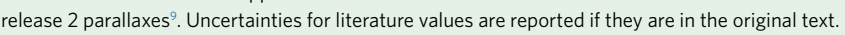

than plasma emission ${ }^{20,21}$. Because the emission is inherently narrowband, the observed broadband emission must be the aggregate emission from regions of different magnetic field strengths within the emitter. The size of a flaring coronal loop that can accommodate such a region is comparable to or larger than the size of GJ 1151 (Methods). This provides additional evidence in support of global magnetospheric currents as the driver of emission as opposed to impulsively heated thermal plasma in compact coronal loops.

Owing to the high electron density in a stellar corona (compared with planetary magnetospheres), an impediment to an auroral cyclotron maser interpretation is the gyro-resonant absorption by ambient thermal electrons at harmonics of the cyclotron frequency ${ }^{11,21}$. Escaping radiation is obtained at coronal densities lower than $\sim 10^{3} \mathrm{~cm}^{-3}$ and $\sim 10^{6} \mathrm{~cm}^{-3}$ for emission at the fundamental and second harmonic, respectively (Methods). These values are orders of magnitude lower than typical coronal densities of solar-type stars ( $\mathrm{F}$ and $\mathrm{G}$ dwarfs) and highly active flare stars ${ }^{19}$. The coronae of X-ray dim quiescent $\mathrm{M}$ dwarfs, however, can have substantially lower base density, and pressure scale heights allowing for escape conditions to be met at heights of $1-3 R_{*}$, where magnetospheric cyclotron maser emission is expected to originate. For example, adopting the empirically determined universal scaling laws for coronal parameters ${ }^{22}$, and assuming a hydrostatic corona, we find that the escape conditions can be met in GJ 1151 at a radius of $2 R_{*}$ for coronal temperatures of $T=0.7 \times 10^{6} \mathrm{~K}$ and $T=1.5 \times 10^{6} \mathrm{~K}$ for fundamental and harmonic emission, respectively (Methods). The escape criterion may also explain why analogous centimetre-wavelength auroral emission has previously been detected in ultracool dwarfs ${ }^{17,18}$ but not in hotter main-sequence stars. Emission at centimetre wavelengths requires a kilogauss-level magnetic field, which is only expected close to the stellar surface where the high electron density may prevent radiation escape in main-sequence stars.

Auroral cyclotron maser emission is powered by persistent acceleration of magnetically confined electrons to $\sim 10 \mathrm{keV}-1 \mathrm{MeV}$ energies. In substellar objects with largely neutral atmospheres the currents are thought to be driven by two processes: (1) breakdown of rigid co-rotation of magnetospheric plasma with the object's magnetic field either due to radial diffusion of outflowing plasma ${ }^{23}$, or interaction between a rotating magnetosphere and the interstellar medium ${ }^{24}$, and (2) sub-Alfvénic interaction of the object's magnetosphere with an orbiting body ${ }^{3,5-7}$. Co-rotation breakdown seen in Jupiter and ultracool dwarfs, which are largely observed to have rotation periods less than $\sim 3 \mathrm{~h}$, is rotation powered and has been shown to generate a radio power of $\sim 10^{13} \mathrm{ergs} \mathrm{s}^{-1} \mathrm{~Hz}^{-1}$ (refs. ${ }^{25,18}$ ). GJ 1151 has an $\sim 3,000 \mathrm{~h}$ rotation period. Assuming coronal parameters comparable to radio-loud ultracool dwarfs, any co-rotation breakdown in GJ 1151 will generate a polar flux that is roughly three orders of magnitude weaker than the observed radio power of $4.3 \times 10^{13} \mathrm{ergs} \mathrm{s}^{-1} \mathrm{~Hz}^{-1}$.

The failure of the co-rotation breakdown model points to a subAlfvénic interaction as the cause of the observed radio emission. This scenario is a scaled-up version of the well known Jupiter-Io electrodynamic engine, and has been proposed as an avenue to study star-planet interaction ${ }^{4-6}$. We checked the feasibility of this scenario by comparing theoretical estimates of the starward Poynting flux with that implied by the brightness of the observed emission. We considered an interaction with an Earth-like planet due to the known preponderance of such planets around red dwarf $\operatorname{stars}^{26}$. A planet in a one- to five-day-long orbit can satisfy the total energy and brightness temperature requirements for the observed radio emission (Methods and Fig. 3).

In the sub-Alfvénic interaction scenario, although an exoplanet is implicated in the radio emission process, we have implicitly assumed that the site of emission is GJ 1151's corona. However, a sizeable fraction of the Poynting flux intercepted by the planet can also dissipate in its magnetosphere ${ }^{4,5}$. As such, the radio emission may have originated in the putative planet's magnetosphere. Recent analysis of optical signatures of star-planet interaction in shortperiod systems suggest that the magnetic fields of some gas-giant planets can be strong enough to generate radio emission at our observation frequency ${ }^{27}$. We note, however, that terrestrial planets, which are more commonly found around $\mathrm{M}$ dwarfs, are expected to have much weaker magnetic fields ${ }^{6}$.

The quiescent nature of GJ 1151 motivated us to study the phenomenology and mechanism of emission and arrive at the starplanet interaction hypothesis. Previous metre-wave observations have almost exclusively focused on highly active stars ${ }^{2,13}$ making it difficult to discern possible star-planet interaction signatures with canonical stellar activity. We suggest that regardless of stellar activity level, detection of periodicity in the radio emission from GJ 1151 at a period distinct from the stellar rotation period can be used to conclusively implicate an exoplanet in the emission process with future observations. The radio-derived periodicity in such systems can additionally be corroborated against the anticipated stellar radial velocity signature. For example, our benchmark model (Earth-mass planet in an approximately one- to five-day orbit) implies a radial velocity signature with semi-amplitude of $\sim 1 \mathrm{~m} \mathrm{~s}^{-1} \times \sin i$, where $i$ is the orbital inclination of the system. Such a radial velocity signature is within the targeted sensitivity of upcoming radial velocity surveys.

We end by noting that our results show that a systematic study of the interaction between stars and short-period exoplanets using their radio emission is feasible. Based on the discovery of GJ 1151 in an $~ 420$-square-degree survey footprint, we expect many tens of such detections from the ongoing LoTSS survey, which will allow a study of star-planet interaction over different stellar types and magneto-ionic interaction regimes.

\section{Methods}

Dynamic spectrum. To produce Fig. 2, the radio data were initially processed with the standard LoTSS processing pipeline ${ }^{8}$, which included direction-dependent instrumental gain and ionospheric corrections. The spectrum was extracted by imaging the field around GJ 1151 using the WSCLEAN software ${ }^{28}$ for the entire $8 \mathrm{~h}$ synthesis in different six equally spaced channels. Similarly, the light curves were obtained over the entire bandwidth by splitting the $8 \mathrm{~h}$ synthesis into six equal parts. The shortest baselines in the LoTSS data have larger levels of systematic errors from mis-subtracted sources. As such, we conservatively chose Briggs' 

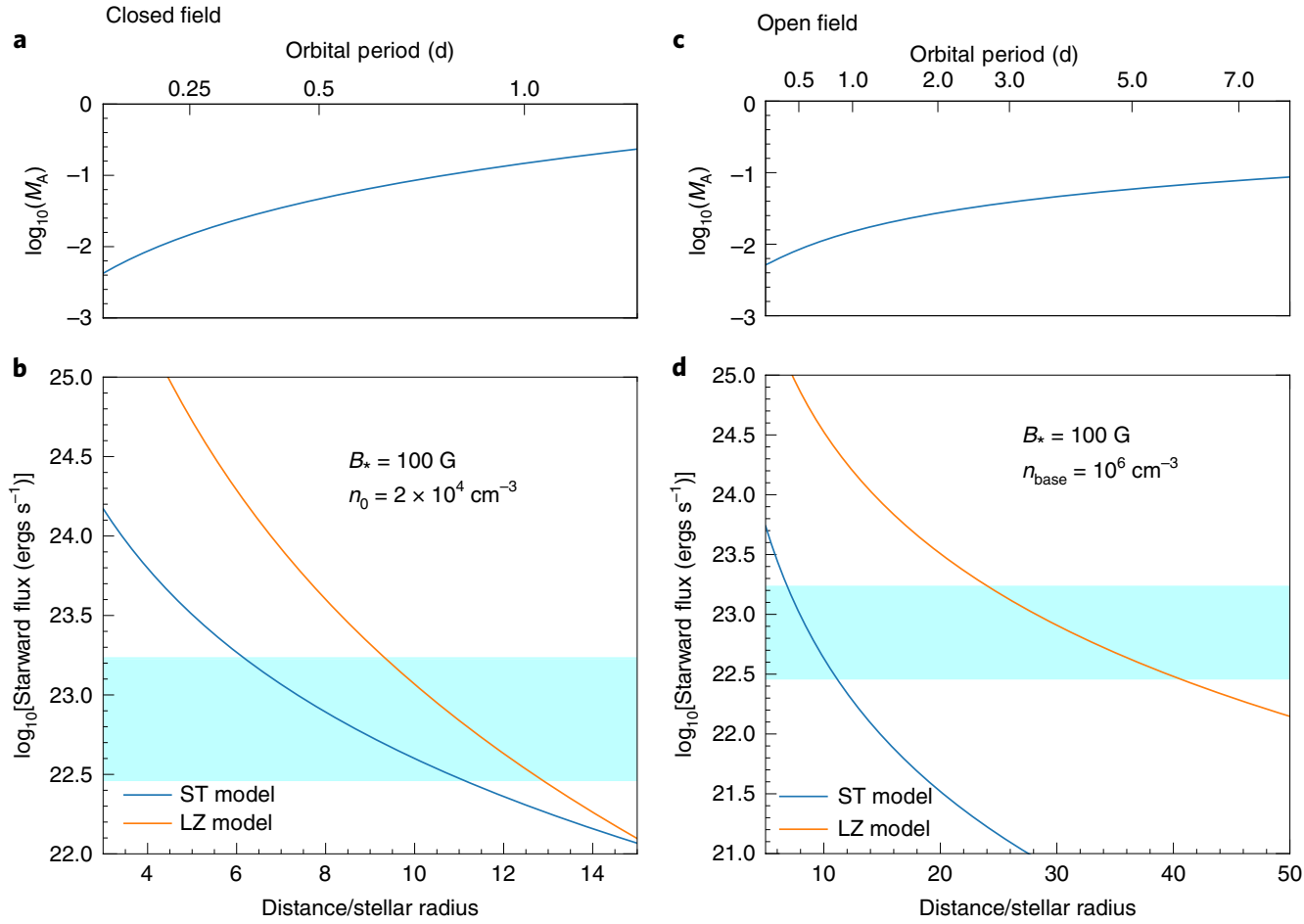

Fig. 3 | A comparison of observationally inferred and theoretical values for the starward Poynting flux from sub-Alfvénic interaction with an Earth-size exoplanet. a-d, A closed dipolar geometry (a,b) and an open Parker spiral geometry (c,d) are assumed for the stellar magnetic field. a,c, Alfvén Mach number of the interaction $\left(M_{A}\right) \cdot \mathbf{b}, \mathbf{d}$, The cyan rectangle is the range allowed by the observed radio flux density. The blue and orange curves show the Poynting flux for two theoretical models of the interaction: the ST model proposed by refs. ${ }^{5,6}$ and the LZ model proposed by ref. ${ }^{7}$. B* is the assumed surface magnetic field of the star. $n_{0}$ is the plasma density at the location of the putative planet (closed-field case) and $n_{\text {base }}$ is the base density of the coronal wind (open-field case). Further details are given in Methods.

weighting with a robustness parameter of -0.5 for the Stokes I images. This leads to higher noise level than naturally weighted images, but is more robust to systematic errors as it down-weights short baselines. Since the Stokes V sky is largely empty, we chose a Briggs' robustness parameter of +0.5 for the Stokes V images, which being closer to natural weighting yields lower noise levels.

Plasma emission. The free energy for plasma emission originates in electron density oscillations, called Langmuir waves, generated by a turbulent injection of impulsively heated plasma ( $T_{1} \sim 10^{8} \mathrm{~K}$ typically) into an ambient colder plasma ( $T \sim 10^{6} \mathrm{~K}$ typically). We used the theoretical expressions for the brightness temperature of plasma emission from ref. ${ }^{19}$. We take the Langmuir wave spectrum to be restricted to a range of wavenumbers: $k_{\min }=2 \pi \nu_{\mathrm{p}} / \nu_{1}$, and $k_{\max }=2 \pi \nu_{\mathrm{p}} /\left(3 v_{\mathrm{e}}\right)$, where $v_{1}$ and $v_{\mathrm{e}}$ are the mean velocities of the hot and cold (ambient) electrons respectively and $\nu_{\mathrm{p}}$ is the plasma frequency. For $k>k_{\max }$, the wave growth is arrested by Landau damping and for $k<k_{\min }$, the waves cannot resonantly exchange energy with the hot electrons. We conservatively take the total energy density in the Langmuir waves to be $10^{-5}$ of the kinetic energy density of the ambient plasma, which is the peak value obtained by both theoretical studies of nonlinear effects and numerical simulations $\mathrm{s}^{29}$. We assume an ambient coronal temperature of $T=2 \times 10^{6} \mathrm{~K}$ which is consistent with the $\mathrm{X}$-ray non-detection of GJ 1151. We assume a hydrostatic density structure with a scale height of

$$
L_{\mathrm{n}} \approx 6 \times 10^{9}\left(T / 10^{6} \mathrm{~K}\right)\left(R_{*} / R_{\odot}\right)^{2}\left(M_{*} / M_{\odot}\right)^{-1} \mathrm{~cm}
$$

where $R_{\odot}$ and $M_{\odot}$ are the solar radius and mass, respectively, and $M_{*}$ is the stellar mass. We varied the hot component temperature from $5 \times 10^{7} \mathrm{~K}$ to $5 \times 10^{8} \mathrm{~K}$ and used equations (15) to (22) from ref. ${ }^{19}$ to calculate the plausible range of brightness temperature for the fundamental and the harmonic. The brightness temperature of the fundamental thus calculated is between $4 \times 10^{9}$ and $2 \times 10^{11} \mathrm{~K}$. The brightness temperature for the harmonic is between $5 \times 10^{11}$ and $1.5 \times 10^{12} \mathrm{~K}$. Even if we assume that the entire stellar disk is filled with continuously flaring coronal loops, then the brightness temperature inferred from the observed flux density is $3.7 \times 10^{12} \mathrm{~K}$. This alone rules out fundamental plasma emission. Even though second harmonic plasma emission can reach $\sim 10^{12} \mathrm{~K}$ brightness temperatures, it suffers from an additional serious problem related to the high degree of polarization observed. Solar harmonic emission has observed polarization levels below about $20 \%$ (ref. ${ }^{29}$ ). The theory allows polarized fractions of up to $\sim 60 \%$ in specific scenarios ${ }^{30}$. However, if coronal loops in the entire stellar disk contribute to the emission, as required by the brightness temperature constraint, then the opposing handedness of emission from regions with oppositely directed magnetic fields must lead to a substantially lower degree of net polarization.

Cyclotron maser from flaring coronal loop. We consider a compact magnetic loop in the stellar corona where impulsively heated thermal plasma is injected and an unstable loss-cone distribution is set up by magnetic mirroring on either ends of the loop. For a continuously operating maser, the brightness temperature is given by ${ }^{21}$

$$
T_{\mathrm{b}}=\frac{m_{\mathrm{e}} v_{0}^{2}}{4 \pi k_{\mathrm{B}}} \frac{\lambda^{2}}{L r_{0}} \approx 2 \times 10^{14}\left(\frac{\beta_{0}}{0.2}\right)^{2}\left(\frac{\lambda}{200 \mathrm{~cm}}\right)^{2}\left(\frac{L}{R_{*}}\right)^{-1} \mathrm{~K}
$$

where $r_{0}$ is the classical electron radius, $L$ is the length scale of the trap, $m_{\mathrm{e}}$ is the electron mass, $k_{\mathrm{B}}$ is the Boltzmann constant, $v_{0}$ is the velocity of the emitting electrons and $\beta_{0}=v_{0} / c$, where $c$ is the speed of light. The emission with the above brightness temperature is centred at the ambient cyclotron frequency and is narrowband: $\Delta \nu / \nu \approx \beta^{2} \alpha_{0}^{2}$, where $\alpha_{0}$ is the opening angle of the loss-cone distribution. The observed broadband emission can be conceptually thought of as an aggregate of $\nu / \Delta \nu$ sites of emission within the magnetosphere. Consider a hypothetical magnetic trap of length $L$ and cross-sectional area of $\pi W^{2} / 4$ (where $W$ is its width). Each site therefore has a projected area of $W L \beta^{2} \alpha_{0}^{2}$. Stellar coronal loops typically have $W<0.1 L$ (ref. ${ }^{31}$ ). We can use these to relate the peak brightness temperature for continuous operation with the observed value to obtain

$$
L \gtrsim 16 R_{*}\left(\frac{\beta}{0.2}\right)^{-4}\left(\frac{\alpha_{0}}{0.5}\right)^{-2}
$$

Even for a high value of $\beta=0.4$, which corresponds to a plasma temperature of $\sim 10^{9} \mathrm{~K}$, we get $L \gtrsim R_{*}$. This suggests that impulsively heated thermal plasma in a compact flaring coronal loop cannot account for the observed brightness temperature.

Escape of cyclotron maser emission. Cyclotron maser emission must necessarily propagate through regions of decreasing magnetic field, where fundamental emission can suffer absorption at the second and higher harmonics. Fundamental 
cyclotron emission is in the $x$-mode, for which the optical depth is at the $s$ th harmonic is ${ }^{21}$

$$
\tau_{s}=\left(\frac{\pi}{2}\right)^{5 / 2} \frac{2}{c} \frac{\nu_{\mathrm{p}}^{2}}{\nu} \frac{s^{2}}{s !}\left(\frac{s^{2} \beta^{2}}{2}\right)^{s-1} L_{\mathrm{B}}
$$

where $\nu_{\mathrm{c}}$ is the ambient cyclotron frequency and $\beta \approx\left(k_{\mathrm{B}} T / m_{\mathrm{e}}\right)^{1 / 2} / c$ is the electron thermal velocity normalized to the speed of light. Equation (4) must be evaluated at $\nu=s \nu_{\mathrm{c}}$. The length scale of integration is the magnetic scale height $L_{\mathrm{B}}=B|\Delta B|^{-1}$ which we take to be of the order of the stellar radius, $\sim 10^{10} \mathrm{~cm}$. We assume a hydrostatic corona close to the star, with radial density evolution of

$$
n(R)=n_{\mathrm{b}} \exp \left[-\frac{R_{*}}{L_{\mathrm{n}}}\left(1-\frac{R_{*}}{R}\right)\right]
$$

where $n_{\mathrm{b}}$ is the base density and the scale height $L_{\mathrm{n}}$ can be computed from the coronal temperature. Both of these are not observationally accessible in X-ray non detected stars such as GJ 1151. We related the density and coronal temperature with empirically determined relationships seen in solar and stellar coronae ${ }^{22}$ : $n=4.3 \times 10^{6}\left(T / 10^{6} \mathrm{~K}\right)^{4.2} \mathrm{~cm}^{-3}$. With this, the absorption coefficients can be computed for any coronal height once the temperature is specified.

Radio emission from sub-Alfvénic interaction. Energetics. A theoretical estimate of the starward Poynting flux due to the star-planet interaction is given by refs. ${ }^{4-7}$ (in c.g.s units)

$$
S_{\text {poynt }}^{\text {th }}=\left[\frac{1}{2} R_{\text {eff }}^{2} v_{\text {rel }} B^{2}\right] \epsilon
$$

where the term in the square brackets is the incident Poynting flux on the planet and $\varepsilon$ captures efficiency factors related to the precise nature of the electrodynamic interaction (details below). $B, R_{\text {eff }}$ and $v_{\text {rel }}$ are, respectively, the stellar magnetic field at the location of the planet, the effective radius of the planetary obstacle and the relative velocity between the stellar wind flow and the planet. In convenient units, we have

$$
S_{\text {poynt }}^{\text {th }} \approx 1.8 \times 10^{22}\left(\frac{R_{\text {eff }}}{6,000 \mathrm{~km}}\right)^{2}\left(\frac{v_{\text {rel }}}{100 \mathrm{~km} \mathrm{~s}^{-1}}\right)\left(\frac{B}{1 \mathrm{G}}\right)^{2}\left(\frac{\epsilon}{0.01}\right) \mathrm{ergs} \mathrm{s}^{-1}
$$

We can compare $S_{\text {poynt }}^{\text {th }}$ to the Poynting flux inferred from the observed radio emission, $S_{\text {poynt }}^{\text {obs }}$, as follows. If $\Delta \nu_{\text {tot }}$ is the total bandwidth of radio emission, $\Omega$ is the beam solid angle of the radio emission, $D$ is the distance to the star and $F$ is the observed flux density, then the total emitted radio power is $P_{\mathrm{em}}=F \Omega D^{2} \Delta \nu_{\text {tot. }}$. We equate the total bandwidth to the peak cyclotron frequency in the star's magnetosphere: $\Delta \nu \approx 2.8 B_{*} \mathrm{MHz}$, where $B_{*}$ is the polar surface magnetic field strength of the star. The observationally inferred starward Poynting flux is then $S_{\text {poynt }}^{\text {obs }}=P_{\text {em }} / \epsilon_{\text {rad }}$, where $\varepsilon_{\text {rad }}$ is the efficiency with which the Poynting flux is converted to cyclotron maser emission. For the case of GJ 1151, $F=0.9 \mathrm{mJy}$ and $D=8.04 \mathrm{pc}$, which gives

$$
S_{\text {poynt }}^{\text {obs }} \approx 1.47 \times 10^{22}\left(\frac{B_{*}}{100 \mathrm{G}}\right)\left(\frac{\Omega}{0.1 \mathrm{sr}}\right)\left(\frac{\epsilon_{\mathrm{rad}}}{0.01}\right)^{-1} \mathrm{ergs} \mathrm{s}^{-1}
$$

Equations (8) and (9) provide a quick check of the feasibility of the star-planet interaction model. A more detailed specification of the various free parameters is given below.

(1) Field topology. We consider two possible magnetic topologies at the location of the planet: a closed-field geometry modelled as a dipole (planet-like) and an open-field geometry that follows a Parker spiral (star-like). These correspond to the left and right panels of Fig. 3, respectively. The observed emission frequency requires the surface field strength of the emitter to be $\gtrsim 60 \mathrm{G}$ and $\gtrsim 30 \mathrm{G}$ for emission at the fundamental and harmonic, respectively. The actual field strength of GJ 1151 cannot be predicted accurately based on available data. We therefore assume $100 \mathrm{G}$ as a benchmark value. We note that this is broadly consistent with GJ 1151's X-ray luminosity and rotation period (see, for example, Figs. 2 and 3 of ref. ${ }^{32}$ ).

(2) Nature of interaction. For both the open- and closed-field cases, we consider two models to specify the interaction efficiency ( $\varepsilon$ in equations (7) and (8)): (1) one from refs. ${ }^{5,6}$, called the ST model hereafter and (2) one proposed in ref. ${ }^{7}$, called the LZ model hereafter. These correspond to the blue and orange lines in Fig. 3, respectively. For the ST model, $\epsilon=M_{\mathrm{A}} \alpha^{2} \sin ^{2} \Theta$, where $M_{\mathrm{A}}$ is the Alfvén Mach number at the planet, $\Theta$ is the angle between the stellar magnetic field at the planet and the stellar wind velocity in the frame of the planet, and $\alpha$ is the relative strength of the sub-Alfvénic interaction. We assume that the planet has a highly conductive atmosphere for which $\alpha=1$. For the LZ model, $\epsilon=\gamma / 2$, where $0<\gamma<1$ is a geometric factor ${ }^{7}$. We assume the average value of $\gamma=0.5$.

(3) Plasma density and velocity. For the open-field case, we assume a base coronal density of $n_{\text {base }}=10^{6} \mathrm{~cm}^{-3}$, which satisfies the radiation escape condition. Because the coronal plasma thermally expands along the openfield lines, we let the base density evolve with radial distance $\propto r^{-2}$. The wind speed is assumed to follow the Parker solution with a base temperature of $10^{6} \mathrm{~K}$. The wind speed dominates the relative velocity in the open-field case. For the closed-field case, there is no substantial stellar wind at the planet's location. Owing to the slow rotation of GJ 1151, the relative velocity is largely determined by the orbital motion of the planet. We assume a constant density of $n_{0}=2 \times 10^{4} \mathrm{~cm}^{-3}$ at the orbital location of the planet. For comparison, the plasma density at Io's orbit is about tens times smaller and is primarily due to Io's volcanic outgassing with negligible contribution from Jupiter itself. We have heuristically assumed a larger value as it can accommodated the presence of a tenuous stellar corona as well as an outgassing planet that is much larger than Io. In our calculation of the Alfvén Mach number, we assume a hydrogen plasma.

(4) Planetary parameters. Owing to the preponderance of Earth-like planets around $\mathrm{M}$ dwarfs, we take the planetary radius to be $6,400 \mathrm{~km}$ and dipolar magnetic field with a surface strength of $1 \mathrm{G}$. The effective radius of a magnetized planet for electrodynamic interaction, $R_{\mathrm{eff}}$, is determined by pressure balance between the planet's magnetosphere and the stellar wind flow. Following ref. ${ }^{5}$, we take this to be the radial distance from the planet at which the planetary and stellar magnetic fields are equal, times a factor of order unity that depends on the angle between the planetary magnetic moment and the stellar field, $\theta_{\mathrm{M}}$. Again following ref. ${ }^{5}$, we take this factor to be 1.46 and 1.73 for the open- and closed-field cases, respectively. These correspond to $\theta_{\mathrm{M}}=\pi / 2$ and $\theta_{\mathrm{M}}=0$, respectively.

(5) Radiation efficiency. This factor depends on the precise nature of the electron momentum distribution, which is not observationally accessible. We therefore take guidance from numerical calculations. Early calculations of the cyclotron maser instability yielded efficiencies of about $1 \%$ (ref. ${ }^{33}$ ). More recent and advanced calculations yielded efficiences of $10 \%$ (ref. ${ }^{34}$ ) or higher. We therefore adopt a range between 1 and $10 \%$.

(6) Beaming angle. The total beam solid angle of the emission cone is necessary to convert emitter power to observed power. We assume an emission cone with half-opening angle $\theta$ and angular width $\Delta \theta$, which are related to the speed of the emitting electrons according to ${ }^{21}: \cos \theta \approx \Delta \theta \approx \beta$. We assume $\beta$ to line in the range $[0.3,0.7]$ corresponding to energies between 20 and $200 \mathrm{keV}$. The beam solid angle then lies between 0.143 and $0.245 \mathrm{sr}$.

With the above prescription, the theoretically expected starward Poynting flux and the observationally inferred values can be computed and contrasted as in Fig. 3.

Brightness temperature. The emitting electrons powered by a star-planet interaction are largely restricted to the stellar flux tube that threads the planet. Assuming a dipolar geometry, the footprint of the flux tube on the star is an ellipse with a semimajor and semi-minor axis of

$$
X=\left(\frac{R_{*}}{d_{\mathrm{p}}}\right)^{3 / 2} R_{\mathrm{p}}
$$

and

$$
Y=X\left(4-\frac{3 R_{*}}{d_{\mathrm{p}}}\right)^{-1 / 2}
$$

respectively. Here $d_{\mathrm{p}}$ is the orbital radius of the planet and $R_{\mathrm{p}}$ is its effective radius. The emitting region has an approximate length of order $R_{\star}$ and we take the geometric mean $2 \sqrt{X Y}$ as its cross-sectional width. For our benchmark model of $R_{\mathrm{p}}=6,400 \mathrm{~km}$ and $d=12.5 R_{*}$, the total area of the emitter normalized to the GJ 1151's projected area is $A /\left(\pi R_{*}^{2}\right) \approx 0.00051$. The area of a single coherent maser site is $A \times \nu / \Delta \nu$ and the observed brightness temperature becomes $T_{\mathrm{b}}=7 \times 10^{15}(\Delta \nu / \nu)^{-1}$. We assume a fractional bandwidth of 0.1 corresponding to $\beta=0.5$, which leads to an intrinsic maser brightness temperature of $\sim 3 \times 10^{16} \mathrm{~K}$. Continuously operating masers of such high brightness temperatures can be driven by a horseshoe or shell-type electron distribution and are known to occur in magnetospheric aurorae in planets ${ }^{35}$.

Duration and duty ratio. Based on one detection in four exposures, the duty ratio of emission is $\sim 0.25$. Because the emission lasts for $>8 \mathrm{~h}$, the orbital period of the planet must be larger than $\sim 1 \mathrm{~d}$. Unlike the Jupiter-Io interaction, which is seen from a special viewpoint (the ecliptic), the range of planetary phases with visible emission is difficult to predict because it depends on (1) the inclination of the orbit, (2) magnetic obliquity and (3) the emission cone opening angle and thickness (which in turn depend on $\beta$ ), which are all unknown. In addition, the source was discovered in $8 \mathrm{~h}$ exposure images from a blind survey. Our detections are therefore biased towards systems where the above factors conspire to yield a longer duration and duty cycle of visible emission than is prototypical.

\section{Data availability}

Source data for Fig. 2 are provided with the paper. The raw LOFAR data are available for download from the LOFAR long-term archive at https://lta.lofar.eu. GJ 1151 was detected in an exposure with observation i.d. L231631. Pre-calibrated 
and in-field source subtracted visibility data (about $50 \mathrm{~GB}$ ) are available from the corresponding author upon reasonable request.

\section{Code availability}

The raw interferometric data were processed with publicly available packages (see ref. ${ }^{8}$ for details). Custom scripts used in the star-planet interaction calculations/ plots are available at https://github.com/harishved/GJ1151_SPI

Received: 22 February 2019; Accepted: 6 January 2020;

Published online: 17 February 2020

\section{References}

1. Schwenn, R. Space weather: the solar perspective. Living Rev. Sol. Phys. 3, $2(2006)$

2. Lynch, C. R., Lenc, E., Kaplan, D. L., Murphy, T. \& Anderson, G. E. $154 \mathrm{MHz}$ detection of faint, polarized flares from UV Ceti. Astrophys. J. Lett. 836, L30 (2017).

3. Zarka, P. Auroral radio emissions at the outer planets: observations and theories. J. Geophys. Res. 103, 20159-20194 (1998).

4. Zarka, P. Plasma interactions of exoplanets with their parent star and associated radio emissions. Planet. Space Sci. 55, 598-617 (2007).

5. Saur, J., Grambusch, T., Duling, S., Neubauer, F. M. \& Simon, S. Magnetic energy fluxes in sub-Alfvénic planet star and moon planet interactions. Astron. Astrophys. 552, A119 (2013).

6. Turnpenney, S., Nichols, J. D., Wynn, G. A. \& Burleigh, M. R. Exoplanetinduced radio emission from M dwarfs. Astrophys. J. 854, 72 (2018).

7. Lanza, A. F. Stellar coronal magnetic fields and star-planet interaction. Astron. Astrophys. 505, 339-350 (2009).

8. Shimwell, T. W. et al. The LOFAR Two-Metre Sky Survey-II. First data release. Astron. Astrophys. 622, A1 (2019).

9. Gaia Collaboration Gaia data release 2. Summary of the contents and survey properties. Astron. Astrophys. 616, A1 (2018).

10. Callingham, J. R., Vedantham, H. K., Pope, B. J. S. \& Shimwell, T. W. LoTSS-HETDEX and Gaia: blind search for radio emission from stellar systems dominated by false positives. Res. Notes Am. Astron. Soc. 3, 37 (2019).

11. Dulk, G. A. Radio emission from the sun and stars. Annu. Rev. Astron. Astrophys. 23, 169-224 (1985).

12. Jackson, P. D., Kundu, M. R. \& White, S. M. Quiescent and flaring radio emission from the flare stars AD Leonis, EQ Pegasi, UV Ceti, Wolf 630, YY Geminorum and YZ Canis Minoris. Astron. Astrophys. 210, 284-294 (1989)

13. Villadsen, J. \& Hallinan, G. Ultra-wideband detection of 22 coherent radio bursts on M dwarfs. Astrophys. J. 871, 214 (2019).

14. Hughes, V. A. \& McLean, B. J. Radio emission from FK Comae. Astrophys. J. 313, 263 (1987)

15. Umana, G., Trigilio, C. \& Catalano, S. Radio emission from Algol-type binaries. I. Results of 1992-1993 VLA survey. Astron. Astrophys. 329, 1010-1018 (1998).

16. Mercier, C., Subramanian, P., Chambe, G. \& Janardhan, P. The structure of solar radio noise storms. Astron. Astrophys. 576, A136 (2015).

17. Hallinan, G. et al. Confirmation of the electron cyclotron maser instability as the dominant source of radio emission from very low mass stars and brown dwarfs. Astrophys. J. 684, 644-653 (2008).

18. Hallinan, G. et al. Magnetospherically driven optical and radio aurorae at the end of the stellar main sequence. Nature 523, 568-571 (2015).

19. Stepanov, A. V. et al. Microwave plasma emission of a flare on AD Leo. Astron. Astrophys. 374, 1072-1084 (2001).

20. Wu, C. S. \& Lee, L. C. A theory of the terrestrial kilometric radiation. Astrophys. J. 230, 621-626 (1979).

21. Melrose, D. B. \& Dulk, G. A. Electron-cyclotron masers as the source of certain solar and stellar radio bursts. Astrophys. J. 259, 844-858 (1982).

22. Peres, G., Orlando, S. \& Reale, F. Are coronae of late-type stars made of solarlike structures? The X-ray surface flux versus hardness ratio diagram and the pressure-temperature correlation. Astrophys. J. 612, 472-480 (2004)

23. Cowley, S. W. H. \& Bunce, E. J. Origin of the main auroral oval in Jupiter's coupled magnetosphere-ionosphere system. Planet. Space Sci. 49, 1067-1088 (2001).

24. Turnpenney, S., Nichols, J. D. \& Wynn, G. A. et al. Auroral radio emission from ultracool dwarfs: a jovian model. Mon. Not. R. Astron. Soc. 470, 4274-4284 (2017).

25. Nichols, J. D., Burleigh, M. R. \& Casewell, S. L. et al. Origin of electron cyclotron maser induced radio emissions at ultracool dwarfs: magnetosphereionosphere coupling currents. Astrophys. J. 760, 59 (2012).

26. Dressing, C. D. \& Charbonneau, D. The occurrence of potentially habitable planets orbiting $\mathrm{M}$ dwarfs estimated from the full Kepler dataset and an empirical measurement of the detection sensitivity. Astrophys. J. 807, 45 (2015).

27. Cauley, P. W., Shkolnik, E. L. \& Llama, J. et al. Magnetic field strengths of hot Jupiters from signals of star-planet interactions. Nat. Astron. 3, 1128-1134 (2019).
28. Offringa, A. R., McKinley, B. \& Hurley-Walker, N. et al. WSCLEAN: an implementation of a fast, generic wide-field imager for radio astronomy. Mon. Not. R. Astron. Soc. 444, 606-619 (2014).

29. Benz, A. O. Plasma Astrophysics: Kinetic Processes In Solar and Stellar Coronae 184 (Kluwer, 1993).

30. Melrose, D. B., Dulk, G. A. \& Gary, D. E. Corrected formula for the polarization of second harmonic plasma emission. Proc. Astron. Soc. Aust. 4, 50-53 (1980).

31. López Fuentes, M. C., Klimchuk, J. A. \& Démoulin, P. The magnetic structure of coronal loops observed by TRACE. Astrophys. J. 639, 459-474 (2006).

32. Shulyak, D., Reiners, A. \& Engeln, A. et al. Strong dipole magnetic fields in fast rotating fully convective stars. Nat. Astron. 1, 0184 (2017).

33. Aschwanden, M. J. Relaxation of the loss-cone by quasi-linear diffusion of the electron-cyclotron maser instability in the solar corona. Astron. Astrophys. Suppl. Ser. 85, 1141-1177 (1990).

34. Kuznetsov, A. A. Kinetic simulation of the electron-cyclotron maser instability: relaxation of electron horseshoe distributions. Astron. Astrophys. 526, A161 (2011).

35. Ergun, R. E., Carlson, C. W. \& McFadden, J. P. et al. Electron-cyclotron maser driven by charged-particle acceleration from magnetic field-aligned electric fields. Astrophys. J. 538, 456-466 (2000).

36. Newton, E. R. et al. The Ho emission of nearby M dwarfs and its relation to stellar rotation. Astrophys. J. 834, 85 (2017).

37. Wright, N. J., Newton, E. R., Williams, P. K. G., Drake, J. J. \& Yadav, R. K. The stellar rotation-activity relationship in fully convective $\mathrm{M}$ dwarfs. Mon Not. R. Astron. Soc. 479, 2351-2360 (2018).

38. Sciortino, S., Maggio, A., Favata, F. \& Orlando, S. X-ray spectroscopy of the active dM stars: AD Leo and EV Lac. Astron. Astrophys. 342, 502-514 (1999).

39. Delfosse, X., Forveille, T., Perrier, C. \& Mayor, M. Rotation and chromospheric activity in field M dwarfs. Astron. Astrophys. 331, 581-595 (1998).

40. Irwin, J. et al. On the angular momentum evolution of fully convective stars: rotation periods for field M-dwarfs from the MEarth transit survey. Astrophys. J. 727, 56 (2011).

41. Houdebine, E. R., Mullan, D. J., Paletou, F. \& Gebran, M. Rotation-activity correlations in $\mathrm{K}$ and $\mathrm{M}$ dwarfs: I. Stellar parameters and compilations of $v$ $\sin i$ and $P / \sin i$ for a large sample of late-K and M dwarfs. Astrophys. J. 822, 97 (2016).

42. Morin, J. et al. Large-scale magnetic topologies of mid M dwarfs. Mon. Not. R. Astron. Soc. 390, 567-581 (2008).

\section{Acknowledgements}

H.K.V. and J.R.C. thank D. Melrose, A. Vidotto and P. Zarka for discussions. H.K.V. thanks V. Ravi and G. Hallinan for discussions. The Leiden LOFAR team gratefully acknowledge support from the European Research Council under the European Unions Seventh Framework Programme (FP/2007-2013)/ERC Advanced Grant NEWCLUSTERS-321271. I.S. acknowledges funding from the European Research Council (ERC) under the European Union's Horizon 2020 research and innovation programme under grant agreement number 694513. G.J.W. gratefully acknowledges support of an Emeritus Fellowship from The Leverhulme Trust. S.P.O. acknowledges financial support from the Deutsche Forschungsgemeinschaft (DFG) under grant BR2026/23. M.H. acknowledges funding from the ERC under the European Union's Horizon 2020 research and innovation programme (grant agreement number 772663. This paper is based (in part) on data obtained with the International LOFAR Telescope (ILT). LOFAR is the Low Frequency Array designed and constructed by ASTRON. It has observing, data processing and data storage facilities in several countries, which are owned by various parties (each with their own funding sources), and that are collectively operated by the ILT foundation under a joint scientific policy. The ILT resources have benefited from the following recent major funding sources: CNRS-INSU, Observatoire de Paris and Université d'Orléans, France; BMBF, MIWF-NRW, MPG, Germany; Science Foundation Ireland (SFI), Department of Business, Enterprise and Innovation (DBEI), Ireland; NWO, The Netherlands; The Science and Technology Facilities Council, UK. This work was in part carried out on the Dutch national e-infrastructure with the support of the SURF Cooperative through grants e-infra 160022 and 160152. The LOFAR software and dedicated reduction packages on https://github.com/apmechev/GRID_LRT were deployed on these e-infrastructure by the LOFAR e-infragroup. This research has made use of data analysed using the University of Hertfordshire high-performance computing facility (http://uhhpc.herts.ac.uk/) and the LOFAR-UK computing facility located at the University of Hertfordshire and supported by STFC (ST/P000096/1). This work was performed in part under contract with the Jet Propulsion Laboratory (JPL) funded by NASA through the Sagan Fellowship Program executed by the NASA Exoplanet Science Institute. B.J.S.P. acknowledges being on the traditional territory of the Lenape Nations and recognizes that Manhattan continues to be the home to many Algonkian peoples. We give blessings and thanks to the Lenape people and Lenape Nations in recognition that we are carrying out this work on their indigenous homelands.

\section{Author contributions}

H.K.V. and J.R.C. developed the detection strategy, cross-matched the optical and radio catalogues, and discovered the source. H.K.V. modelled the radio emission and wrote the manuscript. J.R.C. initiated the LOFAR project that led to the discovery of the 
source and contributed to the manuscript. T.W.S. processed the radio data with software developed by members of the LoTSS survey collaboration including C.T. and M.J.H. C.T. wrote the software to extract quick-look dynamic spectra. H.J.A.R. is the principal investigator of the broader LOFAR Two-Metre Sky Survey. All authors commented on the manuscript.

\section{Competing interests}

The authors declare no competing interests.

\section{Additional information}

Extended data is available for this paper at https://doi.org/10.1038/s41550-020-1011-9. Correspondence and requests for materials should be addressed to H.K.V.

Reprints and permissions information is available at www.nature.com/reprints.

Publisher's note Springer Nature remains neutral with regard to jurisdictional claims in published maps and institutional affiliations.

(C) The Author(s), under exclusive licence to Springer Nature Limited 2020 


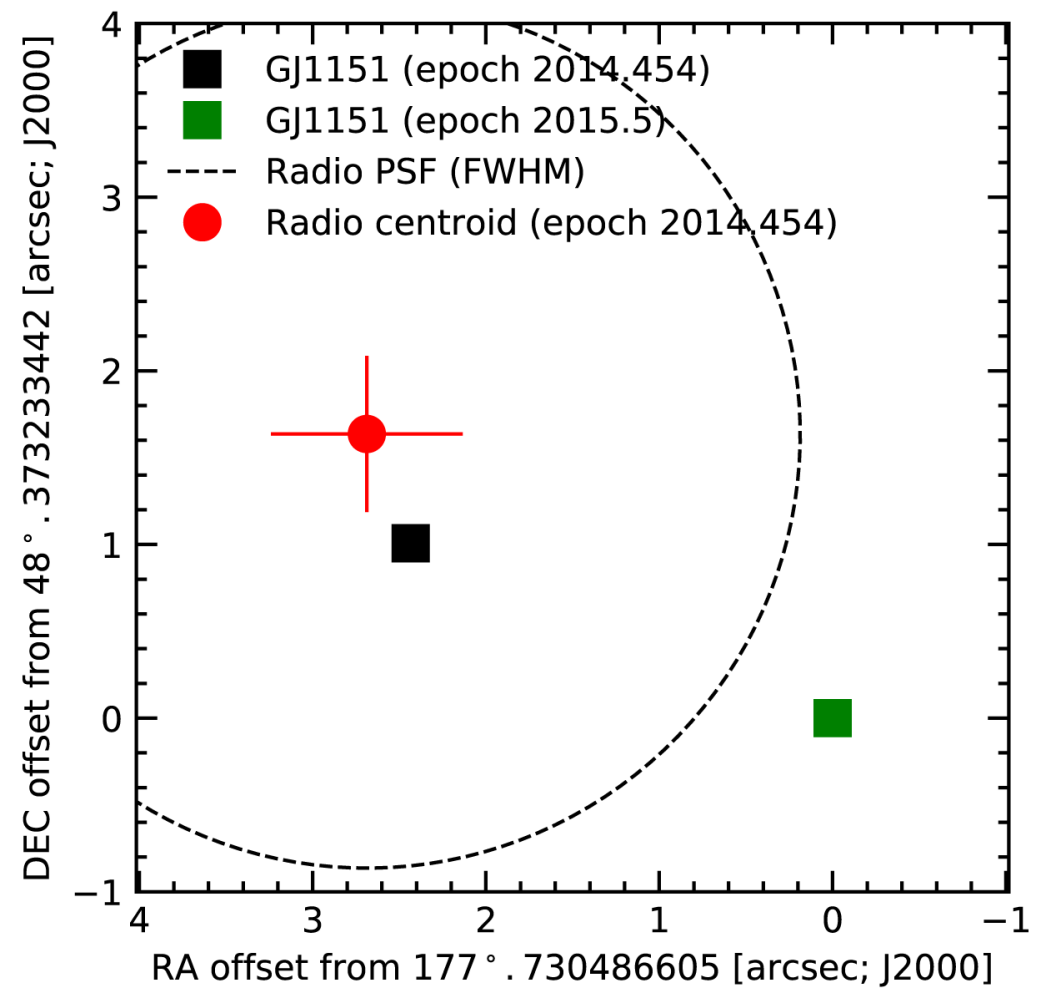

Extended Data Fig. 1 | Astrometric association of the radio source with the star GJ1151. Relative astrometry of the radio source in GJ 1151 and the optical position of M-dwarf star GJ 1151. The optical position and proper-motion correction is based on the Gaia DR2 catalog. The error-bars show the $\pm 1 \sigma$ errors on the radio source centroid that were computed by adding the formal errors in source-finding and the absolute LoTSS astrometric uncertainty in quadrature. 